\title{
Generalizing Neural Branch Prediction
}

\author{
DANIEL A. JIMÉNEZ \\ Rutgers University
}

Improved branch prediction accuracy is essential to sustaining instruction throughput with today's deep pipelines. Traditional branch predictors exploit correlations between pattern history and branch outcome to predict branches, but there is a stronger and more natural correlation between path history and branch outcome. We explore the potential for exploiting this correlation. We introduce piecewise linear branch prediction, an idealized branch predictor that develops a set of linear functions, one for each program path to the branch to be predicted, that separate predicted taken from predicted not taken branches. Taken together, all of these linear functions form a piecewise linear decision surface. We present a limit study of this predictor showing its potential to greatly improve predictor accuracy.

We then introduce a practical implementable branch predictor based on piecewise linear branch prediction. In making our predictor practical, we show how a parameterized version of it unifies the previously distinct concepts of perceptron prediction and path-based neural prediction. Our new branch predictor has implementation costs comparable to current prominent predictors in the literature while significantly improving accuracy. For a deeply pipelined simulated microarchitecture our predictor with a $256-\mathrm{KB}$ hardware budget improves the harmonic mean normalized instructions-per-cycle rate by $8 \%$ over both the original path-based neural predictor and 2Bc-gskew. The average misprediction rate is decreased by $16 \%$ over the path-based neural predictor and by $22 \%$ over $2 \mathrm{Bc}$-gskew.

Categories and Subject Descriptors: C.1.1 [Computer Systems Organization]: Processor Architectures-Single data stream architectures

General Terms: Performance

Additional Key Words and Phrases: Branch prediction, machine learning

ACM Reference Format:

Jiménez, D. A. 2009. Generalizing neural branch prediction. ACM Trans. Architec. Code Optim. 5, 4, Article 17 (March 2009), 27 pages. DOI = 10.1145/1498690.1498692 http://doi.acm.org/ $10.1145 / 1498690.1498692$

This research is supported by National Science Foundation grants CCF-0311091 and CCF-0545898. Author's address: D. Jiménez, Department of Computer Science, The University of Texas at San Antonio, One UTSA Circle, San Antonio, TX 78249-1644; email: djimeneth@gmail.com or djimenez@acm.org

Permission to make digital or hard copies part or all of this work for personal or classroom use is granted without fee provided that copies are not made or distributed for profit or commercial advantage and that copies show this notice on the first page or initial screen of a display along with the full citation. Copyrights for components of this work owned by others than ACM must be honored. Abstracting with credit is permitted. To copy otherwise, to republish, to post on servers, to redistribute to lists, or to use any component of this work in other works requires prior specific permission and/or a fee. Permissions may be requested from the Publications Dept., ACM, Inc., 2 Penn Plaza, Suite 701, New York, NY 10121-0701 USA, fax +1 (212) 869-0481, or permissions@acm.org. (C) 2009 ACM 1544-3566/2009/03-ART17 \$5.00 DOI 10.1145/1498690.1498692 http://doi.acm.org/ $10.1145 / 1498690.1498692$

ACM Transactions on Architecture and Code Optimization, Vol. 5, No. 4, Article 17, Publication date: March 2009. 


\section{INTRODUCTION}

Deeper pipelines improve overall performance by allowing more aggressive clock rates. However, some potential performance is lost due to the resulting increase in branch misprediction latencies. Indeed, branch misprediction latency is the most important component of performance degradation as microarchitectures become more deeply pipelined [Sprangle and Carmean 2002]. Branch predictors must improve to avoid the increasing penalties of mispredictions.

In this article, we introduce piecewise linear branch prediction that works by learning a set of linear functions for each branch that together comprise a piecewise linear surface in a space of branch outcome pattern histories. This surface separates predicted taken branches from predicted not taken branches. A piecewise linear surface allows the predictor to learn the behavior of certain linearly inseparable branches that previous neural predictors were unable to learn [Jiménez and Lin 2002]. Figure 1 shows examples of decision surfaces learned by the perceptron predictor (a) and piecewise linear branch prediction (b) for the linearly inseparable exclusive-OR (XOR) function. The two input variables are represented by the $x$ and $y$ axes, negative for false and positive for true. The output is represented by the color white for false and black for true. XOR cannot be learned by the perceptron predictor, but it is easily learned by piecewise linear branch prediction.

We first describe an idealized version of piecewise linear branch prediction, giving algorithms to explain the concepts but paying no attention to microarchitectural implementation constraints. In the context of a boundless hardware and computational budget, we give the results of a limit study comparing piecewise linear branch prediction to idealized versions of previous prediction mechanisms. We show that piecewise linear branch prediction reduces mispredictions by an average of $16 \%$ over the next best predictor.

We then derive a practical piecewise linear branch predictor suitable for microarchitectural implementation by constraining the state used by the idealized predictor and using ahead-pipelining to mitigate its latency. Using a cycle-accurate microarchitectural simulator, we show that the practical piecewise linear branch predictor improves normalized harmonic mean instructionsper-cycle rate (IPC) by $8 \%$ over both 2Bc-gskew [Seznec et al. 2002] and the path-based neural predictor [Jiménez 2003] for a 256-KB hardware budget. At lower hardware budgets, the new predictor also yields a significant speedup.

\subsection{Contributions}

This article makes the following contributions:

(1) We introduce piecewise linear branch as an idealized algorithm apart from implementation concerns. We show that this predictor yields an average misprediction rate $16 \%$ lower than that given by idealizations of other branch prediction mechanisms. 


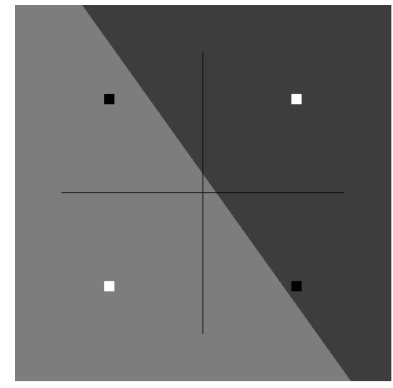

(a)

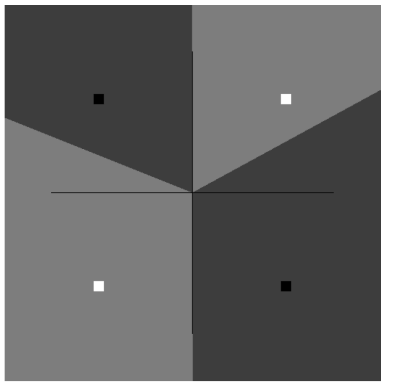

(b)

Fig. 1. The XOR function cannot be learned by a perceptron (a), but can be learned using a piecewise linear decision surface (b).

(2) We show how to constrain the storage and latency used by the algorithm to derive a practical piecewise linear branch predictor suitable for microarchitectural implementation.

(3) We show that constraining our new predictor in certain ways yields algorithms that are equivalent to the perceptron predictor and to path-based neural prediction. Thus, piecewise linear branch prediction conceptually unifies these two previously distinct predictors. Furthermore, we show that perceptron and path-based neural predictors are the two worst-case examples of this generalization in terms of accuracy; thus, the new predictor is strictly superior.

(4) Using a cycle-accurate microarchitectural simulator, we show that the practical version of piecewise linear branch prediction reduces mispredictions by an average of $9 \%$ over path-based neural prediction with a $32-\mathrm{KB}$ hardware budget and by $16 \%$ with a $256-\mathrm{KB}$ hardware budget. Harmonic mean normalized IPC is improved by $8 \%$ over the next best branch predictor at the 256-KB hardware budget.

\subsection{Organization}

In Section 2, we discuss related work in neural branch prediction. In Section 3, we introduce our new predictor. In Section 4, we present a limit study of the new predictor and idealized versions of other predictors. In Section 5, we show how to derive a practical piecewise linear predictor suitable for microarchitectural implementation. In Section 6, we show how this practical predictor exposes the fact that piecewise linear branch prediction is a generalization of neural prediction giving rise to a family of branch predictors with the perceptron predictor and path-based neural branch prediction as two extremes. In Section 7, we discuss our experimental methodology for evaluating the practical piecewise linear predictor. In Section 8, we provide the results of experiments showing the potential for improved accuracy and performance given by our new predictor organization. In Section 9, we provide an analysis of why piecewise linear branch prediction outperforms previously proposed neural branch predictors. Finally, in Section 10, we conclude and provide directions for future research. 


\section{BACKGROUND AND RELATED WORK}

In this section, we discuss related work in branch prediction. The predictor we introduce in this article is an extension of neural branch predictor, so we focus on neural branch prediction research.

\subsection{The Perceptron Predictor}

The perceptron predictor [Jiménez and Lin 2001] uses a simple linear neuron known as a perceptron [Block 1962] to perform branch direction prediction. Perceptrons achieve better accuracy than two-level adaptive branch prediction because of their ability to exploit long history lengths that have been shown to provide additional correlation for branch predictors [Evers et al. 1998]. Another study suggests ways to implement the predictor using techniques from highspeed arithmetic [Jiménez and Lin 2002], but the latency of the predictor is still high. The most accurate single-component branch predictors in the literature are neural branch predictors [Loh and Henry 2002; Jiménez and Lin 2002]. Unfortunately, the high latency of the original perceptron predictor and makes it impractical for improving performance.

\subsection{Research Related to Neural Branch Prediction}

Many studies have extended the perceptron predictor. Loh and Henry [2002] use the perceptron predictor as a component of a larger hybrid predictor. Thomas et al. find salient history bits for the perceptron predictor using dynamic dataflow analysis. Akkary and Srinivasan [2004] adapt the perceptron predictor to provide confidence estimates for speculation control. Intel includes the perceptron predictor in one of its IA-64 simulators for researching future microarchitectures [Brekelbaum et al. 2002]. Falcón et al. [2004] use a perceptron predictor as a component of a prophet/critic hybrid predictor that runs the branch predictor ahead to second-guess previous predictions and possibly reverse them.

Each of these items of related work enhance neural prediction without changing its basic prediction mechanism. In this article, we focus on improving the basic mechanism; we expect that other work such as prophet/critic [Falcón et al. 2004] will be improved by incorporating piecewise linear branch prediction.

\subsection{Neural Branch Prediction Background}

Neural branch predictors keep a table of weights vectors, that is, vectors of small integers that are learned through the perceptron learning rule [Jiménez and Lin 2001; Block 1962]. As in global two-level adaptive branch prediction [Yeh and Patt 1993; McFarling 1993], a shift register records a global history of outcomes of conditional branches, recording true for taken, or false for not taken.

To predict a branch outcome, a weights vector is selected by indexing the table with the branch address modulo the number of weights vectors. The dot product of the selected vector and the global history register is computed, where true in the history represents 1 and false represents -1 . If the dot product is at least 0 , then the branch is predicted taken, otherwise it is predicted not taken. 
A number of arithmetic techniques can be applied to perform the prediction and training computations efficiently [Jiménez and Lin 2002].

\subsection{Path-Based Neural Branch Prediction}

In a recent paper, we describe a neural predictor that achieves lower latency and improved accuracy over previous neural branch predictors [Jiménez 2003] by staggering computations in time, predicting a branch using a weights vector selected dynamically along the path to that branch. The path-based neural branch predictor has improved accuracy over previous neural predictors because it is able to find correlations with path history as well as pattern history. In this article, we present a new neural predictor that achieves even better accuracy at much the same latency.

\subsection{O-GEHL Branch Predictor}

Since the publication of our initial work on piecewise linear branch prediction, Seznec [2005] has presented his Optimized Geometric History Length predictor (O-GEHL). This predictor indexes several tables of counters, each of which has a recent branch history using a different history length. The history lengths for each table form a geometric series. The chosen counters are summed using an adder tree, similar to the perceptron predictor. This strength of this predictor is its ability to represent compactly information from very long histories (e.g., 640 past branches). In the first championship branch prediction competition (CBP), O-GEHL achieved an MPKI of 2.627 [Seznec 2005]. Over the same set of traces, an optimized version of piecewise linear branch prediction achieved 2.742 MPKI [Jiménez 2005].

\section{PIECEWISE LINEAR BRANCH PREDICTION}

This section introduces our new predictor, the piecewise-linear branch predictor. We discuss the intuition behind the idea, then give the algorithm. It is important to note that, at this point, we are presenting an idealized predictor without concern for its implementation since we are only concerned with its predictive power. In Section 5, we derive a practical branch prediction from this idealized predictor.

\subsection{Intuition}

Branch predictors exploit the correlation between the history of a branch and its outcome. One way to conceptually organize the notion of branch history is to consider all of the program paths of a given length $h$ ending in a branch $B$. For our purposes, a path is a dynamic sequence of branches ending in $B$. The identities, positions, and outcomes of such a path usually correlate highly with the outcome of a branch. For each branch $B$, our predictor tracks the elements of every path leading to $B$. The predictor keeps track of the tendency of a given branch in a given position in the history to agree with the outcome of $B$. That is, for every component of every path, the predictor tracks the correlation of that component with the outcome of $B$. To make a prediction, the correlations of each component of the current path are aggregated to form a prediction. 
3.1.1 What is "piecewise linear?". This aggregation is a linear function of the correlations just for the current path. This linear function induces a hyperplane that is used to decide whether to predict taken or not taken: If the global branch outcome pattern history lies on one side of the hyperplane, the branch is predicted taken, otherwise, it is predicted not taken. Since there are many paths leading to $B$, there are many different linear functions used to predict $B$. Taken as a whole, the linear functions used to predict $B$ from a piecewiselinear surface separating paths that lead to predicted taken branches from paths that lead to predicted not taken branches. In machine-learning terminology, such a separating surface is called a decision surface. In Section 6, we will show that piecewise linear branch prediction is a generalization of neural branch prediction [Jiménez and Lin 2001], which uses a single linear function for a given branch, and path-based neural branch prediction [Jiménez 2003], which uses a single global piecewise-linear function to predict all branches. As a perceptron-like algorithm, the predictor finds the sum of weights or their negations based on whether the corresponding history bits represent taken or not taken branches.

\subsection{Description of the Algorithm}

The algorithm has two components: a prediction function and an update procedure. The following variables are used by the algorithm:

$-W$ A three-dimensional array of integers. The indices of this array are the branch address, the address of a branch in the path history, and the position in the history. $W$ keeps track of correlations for every branch in the program. We can think of $W$ as a set of matrices, one for each branch, whose columns correspond to branches in the path history and whose rows correspond to positions in the history. $W[B, 0,0]$ is the weight that keeps track of the tendency of branch $B$ to be taken. This weight is the bias weight for $B$. Addition and subtraction on elements of $W$ saturate at +127 and -128 . The dimensions of the array are arbitrarily large, that is, large enough to accommodate any access that might be made during the algorithm. This fact alone makes the predictor infeasible for actual implementation. Nevertheless, we constrain the storage in our practical version of the predictor presented later.

$-h$ The global history length. This is a small integer.

- GHR The global history register. This vector of bits accumulates the outcomes of branches as they are executed. Branch outcomes are shifted into the first position of the vector. The length of this register is $h$.

- GA An array of addresses. As branches are executed, their addresses are shifted into the first position of this array. Taken together, GHR and GA give the path history for the current branch to be predicted. The length of this array is $h$.

- output An integer. This integer is the value of the linear function computed to predict the current branch.

Figure 2 shows the function predict that computes the Boolean prediction function. The function accepts as a parameter the address of the branch to 


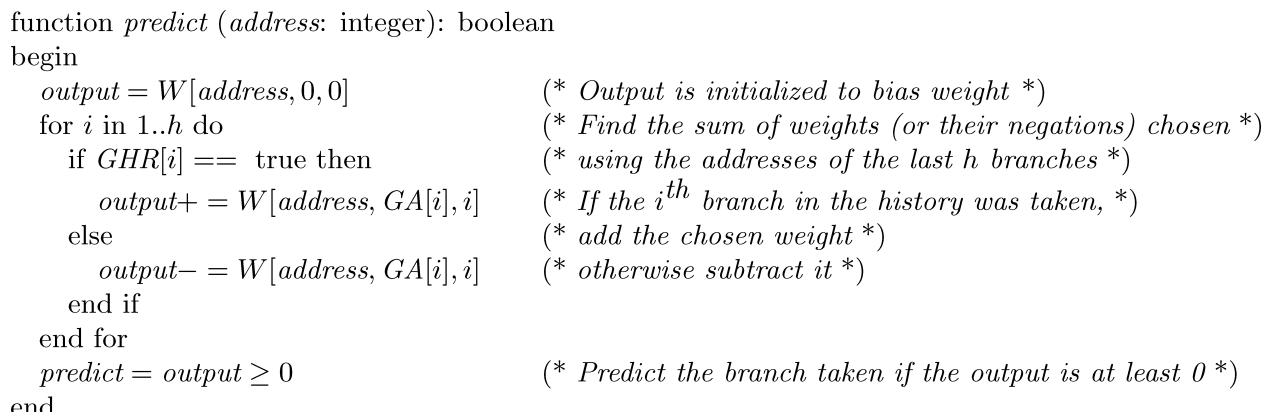

Fig. 2. Prediction algorithm.

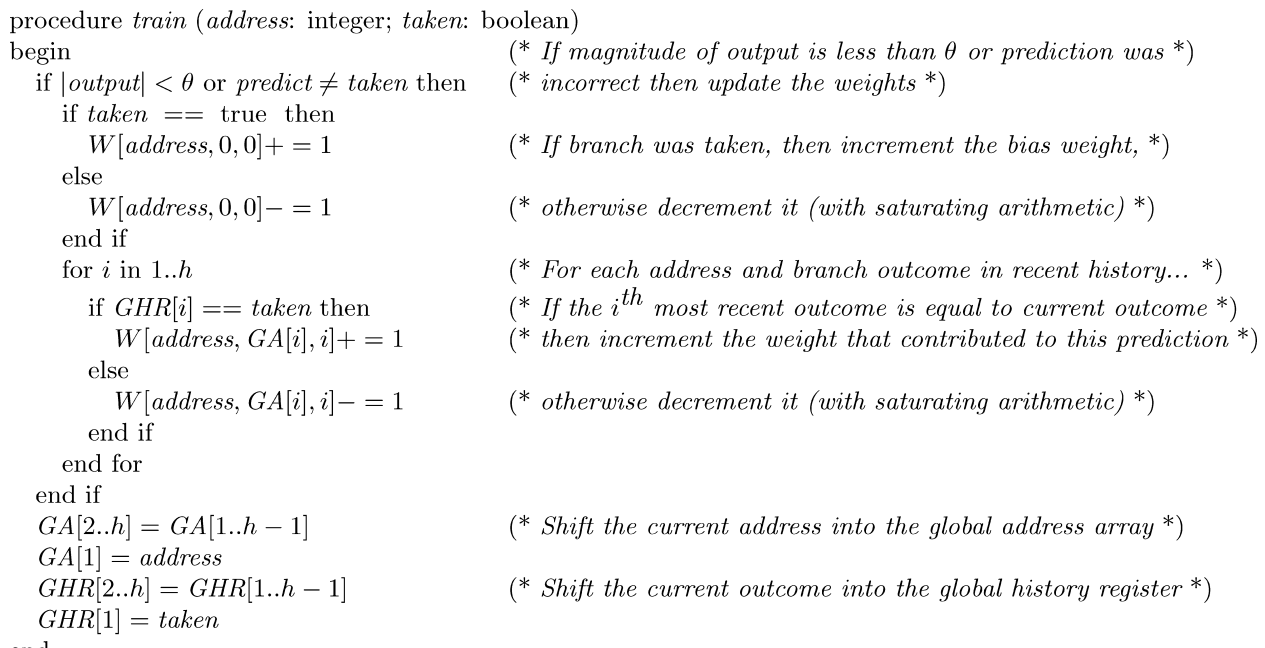

Fig. 3. Training algorithm.

be predicted. The branch is predicted taken if predict returns true, not taken otherwise. Figure 3 shows the procedure train used to update the predictor. It accepts as parameters the address and Boolean outcome of the branch. It assumes that all variables retain the values they had at the end of the invocation of predict for this branch. The training algorithm uses a threshold parameter $\theta$ to decide when to stop updating the predictor; when the magnitude of the output of the predictor exceeds $\theta$, the predictor has learned the behavior of the branch sufficiently well. Without such a threshold parameter, the predictor might overtrain and be slow to respond to changes in branch behavior. This algorithm is similar to other neural predictors, such as the perceptron predictor [Jiménez and Lin 2001] and the path-based neural predictor [Jiménez 2003]. We choose the value of $\theta$ using the same formula from the path-based neural predictor paper, $\theta=2.14(h+1)+20.58$. 


\section{LIMIT STUDY}

When a new predictor is introduced, it is instructive to evaluate its predictive power relative to previous work without regard to implementation concerns, such as area and latency. This section presents a limit study of branch prediction using piecewise linear branch prediction as well as idealized versions of other predictors. We show that our new predictor is able to achieve very low misprediction rates compared with other idealized predictors.

\subsection{Predictors Simulated}

We simulate the following predictors to compare with our new predictor:

4.1.1 2Bc-gskew. We simulate 2Bc-gskew, a hybrid predictor combining a bimodal component with egskew that predicts using the majority of three components: the bimodal predictor and two global history predictors indexed by special hash functions that mitigate destructive interference. A version of this predictor was planned for the Alpha EV8 processor [Seznec et al. 2002]. We have observed that $2 \mathrm{Bc}$-gskew is the most accurate branch predictor based on two-level adaptive prediction in the academic literature.

4.1.2 Perceptron Predictor. We simulate a version of the perceptron predictor that combines global and per-branch history information [Jiménez and Lin 2002]. This predictor has been shown to be more accurate than even the most aggressive multicomponent hybrid predictor [Jiménez and Lin 2002]. Thus, it would be superfluous to compare against other combined global and per-branch hybrid predictors. For this study, a history length of $h$ for the global/local perceptron means that $h$ global and $h$ local history bits are used.

4.1.3 Path-Based Neural Predictor. We simulate the path-based neural predictor [Jiménez 2003]. As in piecewise linear branch prediction, this predictor aggregates weights from the elements of the path to the branch to be predicted, but the weights are shared globally among all branches instead of being associated with a particular branch. This path-based predictor is highly accurate. Thus, we do not include other, less-accurate path-based techniques.

4.1.4 Prediction by Partial Matching. Chen et al. [1996] use the idea of prediction by partial matching (PPM) from data compression to study branch prediction. The predictor is proposed as a theoretically optimal predictor, hence its inclusion in our limit study. A PPM predictor with history length $h$ is a set of $h+1$ Markov predictors, each of which is somewhat similar to a twolevel adaptive branch predictor. The predictors are numbered $0 . . h$ and the $i^{\text {th }}$ predictor uses a history length of $i$. The first predictor tracks the bias of the branch independent of its history. A global history of branch outcomes is kept. In our study, each branch is allocated its own PPM predictor. The current global history is used to find the longest matching entry in one of the $h+1$ Markov predictors, which is used to give the prediction. The predictors are updated with the update exclusion policy used in the original work, that is, only the Markov predictor that made the prediction and any higher-order predictors are updated. 


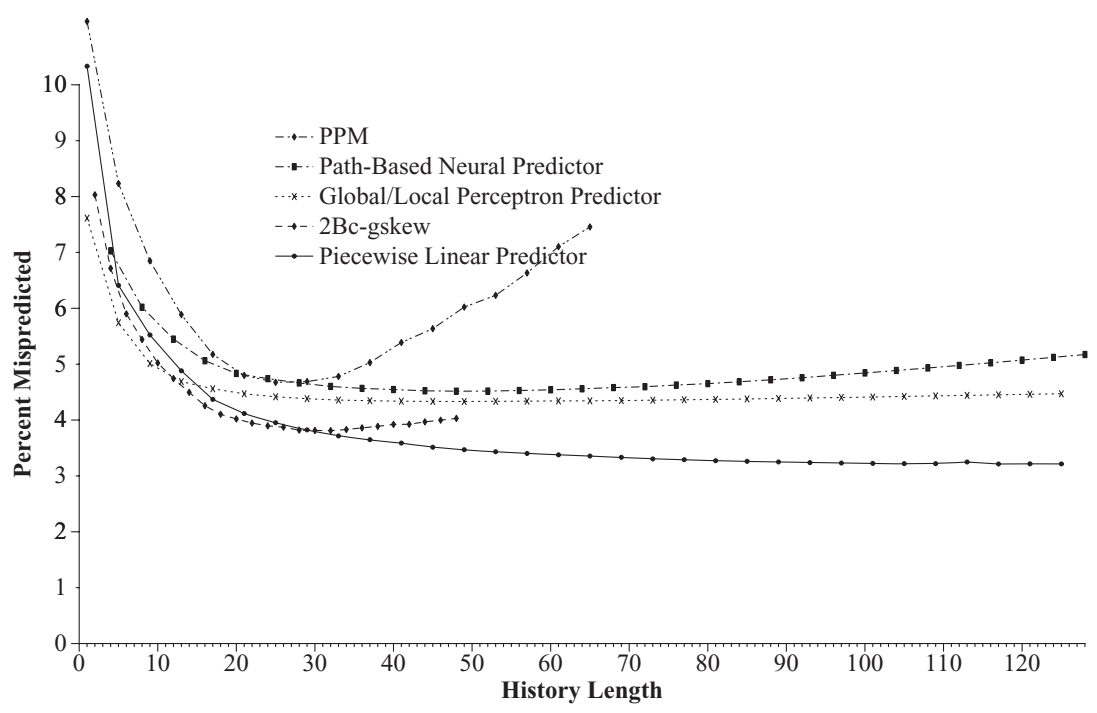

Fig. 4. History lengths versus misprediction rates.

\subsection{Limit Study Methodology}

In this article, we present the results of two sets of experiments. In this section, we present a limit study focused only on accuracy. In Section 8, we present a study of our proposed practical branch predictor in a cycle-accurate microarchitectural framework. For this limit study, we use traces generated by SimpleScalar/Alpha on the same 15 SPEC CPU integer benchmarks we use for Section 8.

To isolate the predictive power of each prediction mechanism from the effects of branch interference, each predictor is allowed to use an arbitrary amount of storage. For the perceptron predictor and path-based neural predictor, one weights vector is allocated to each static branch. For 2Bc-gskew, each table in the predictor is given a virtual 48-bit address space. In other words, the $2 \mathrm{Bc}$-gskew predictor is given a virtual hardware budget of 256 terabytes. The simulation of this budget is facilitated through the use of a data structure that brings a predictor entry into existence only when it is first accessed.

Branch predictor accuracy is highly sensitive to history length [Evers et al. 1998]. We simulate each predictor on all of the traces for a variety of history lengths. For each predictor except for piecewise linear prediction, we simulate a large enough range of history lengths to show that there is a best history length delivering the lowest misprediction rate for that predictor. For piecewise linear prediction, there does not seem to be a best history length; accuracy continues to improve with longer histories.

Figure 4 shows the average misprediction rate for each predictor at various history lengths. For history lengths of up to 30, the 2Bc-gskew mechanism delivers the best accuracy. After that point, piecewise linear branch prediction is the best. 


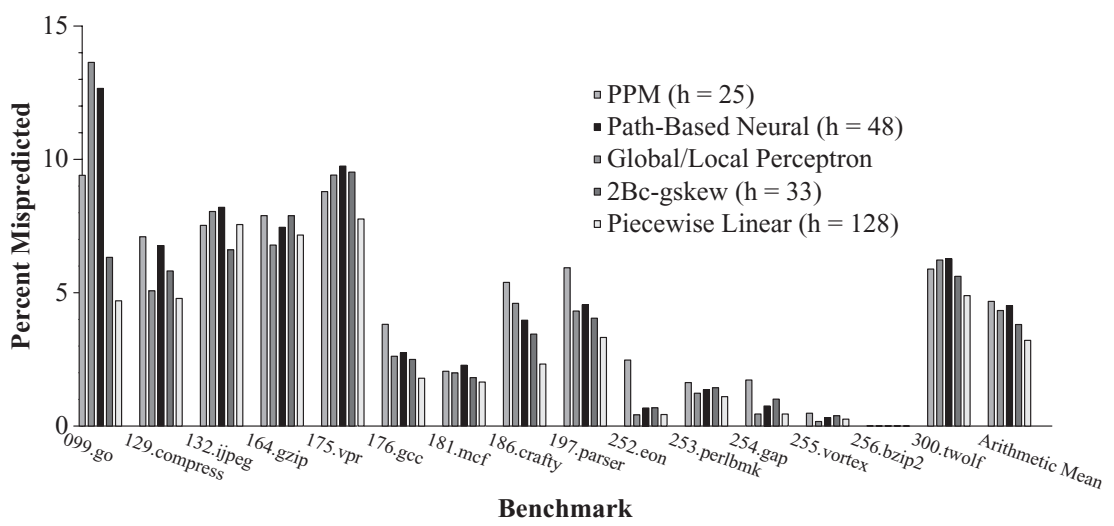

Fig. 5. Misprediction rate per benchmark with the best history for each predictor.

Figure 5 shows the misprediction rate for each benchmark as well as the arithmetic mean. The chart shows the misprediction rates for each predictor using the history length that yields the best average misprediction rate for that predictor. The values of these best history lengths are given in the legend of the graph. Each of these predictors is one of the very best predictors in recent literature. Still, the piecewise linear branch predictor manages to outperform them all. The next best predictor, 2Bc-gskew, gives an average misprediction rate of $3.81 \%$. Piecewise linear branch prediction gives an average misprediction rate of $3.21 \%$, a reduction of $16 \%$. It reduces mispredictions by $26 \%$ over the third most accurate predictor, a global/local perceptron predictor, which yields a misprediction rate of $4.33 \%$. Prediction by partial matching achieves only a $4.67 \%$ misprediction rate, the least accurate of all predictors tested. Piecewise linear branch prediction gives the lowest misprediction rate on 13 out of the 15 benchmarks. It yields the second lowest misprediction rate on the other two benchmarks, and on those two benchmarks two different predictors are the best. Thus, without regard to implementation concerns, piecewise linear branch prediction is a consistently better prediction mechanism than any of the other predictors.

\section{A PRACTICAL PIECEWISE LINEAR BRANCH PREDICTOR}

In this section, we present a practical version of piecewise linear branch prediction with implementation constraints similar to other practical predictors. There are two constraints on a practical predictor that the idealized version presented so far does not satisfy: limited area and limited latency.

\subsection{Limiting Area of the W Array}

The indices of the $W$ array must be limited to keep them from exceeding the practical bounds of an implementable branch predictor. We limit the first two indices by taking them modulo two integers $n$ and $m$. In a realistic implementation, $n$ and $m$ would be chosen as powers of 2 to make the modulo operation 
a simple mask. We limit the third index by choosing an appropriate value $h$ for the history length. Thus, $W$ becomes an $n \times m \times(h+1)$ three-dimensional array of 8-bit weights. Limiting the second array index to $0 . . n-1$ also reduces the bits required to store the $G A$ array since each address can be represented modulo $n$ requiring at most $\log _{2} n+1$ bits.

Of course, limiting the indices of the $W$ matrix will have an adverse impact on the accuracy of the predictor, since weights will be aliased between different paths and different branches. We will show in Section 8 that the piecewise linear predictor still delivers good accuracy despite this aliasing.

\subsection{Limiting Latency of the Prediction}

Computing the output used to predict the branches requires adding $h+1$ numbers each retrieved from different, possibly nonadjacent positions in $W$.

A naive computation of this output would take many cycles because of adder delay and access delay for the memory that comprises $W$. For sufficiently long histories, it might take as many cycles as it takes to resolve the branch being predicted.

We use ahead pipelining to mitigate the latency of this computation. Ahead pipelining means the computation is pipelined and begins before the branch to be predicted is fetched. It has been applied to traditional two-level branch predictors [Jiménez 2002; Seznec and Fraboulet 2003] as well as neural branch predictors [Jiménez 2003; Tarjan et al. 2004]. An ahead-pipelined predictor may only use information it has on hand. In particular, it cannot know which branch it will be used to predict ahead of time. It can, however, use current knowledge of the path to home in on information in its tables that is likely to be of use in making the prediction.

\subsection{An Ahead-Pipelined Piecewise Linear Branch Predictor}

Branch predictions speculatively drive partial computations of the outputs of $h$ future branches. The $W$ array is now an $n \times m \times(h+1)$ three-dimensional array of 8-bit weights. The bias weight for a branch with address $B$ is now kept in $W[b \bmod n, b \bmod m, 0]$, providing a more uniform utilization of the now limited number of weights in $W$. The $W$ array is indexed by the branch address modulo $n$. Since this index is not known ahead of time, the algorithm keeps $n$ copies of the speculative predictor state to drive $n$ possible predictions. Once the branch address becomes known, it is used to select one of the $n$ predictions. Little extra speculative state is required for small values of $n$.

5.3.1 Extra State for the Ahead-Pipelined Predictor. Ahead-pipelining the predictor requires additional state to store intermediate results of computations. $R$ and $S R$ are $n \times h$ two-dimensional arrays of small integers used as intermediate storage for computing the output of the predictor. $S R[i, j]$ holds the speculative partial sum for predicting the $j^{\text {th }}$ branch in the future whose address modulo $n$ is $i$. Extending terminology from the original path-based neural predictor [Jiménez 2003], $S R$ and $R$ are shift matrices composed of $n$ shift vectors. $S R$ is like a queue through which partial sums proceed. Partial sums 


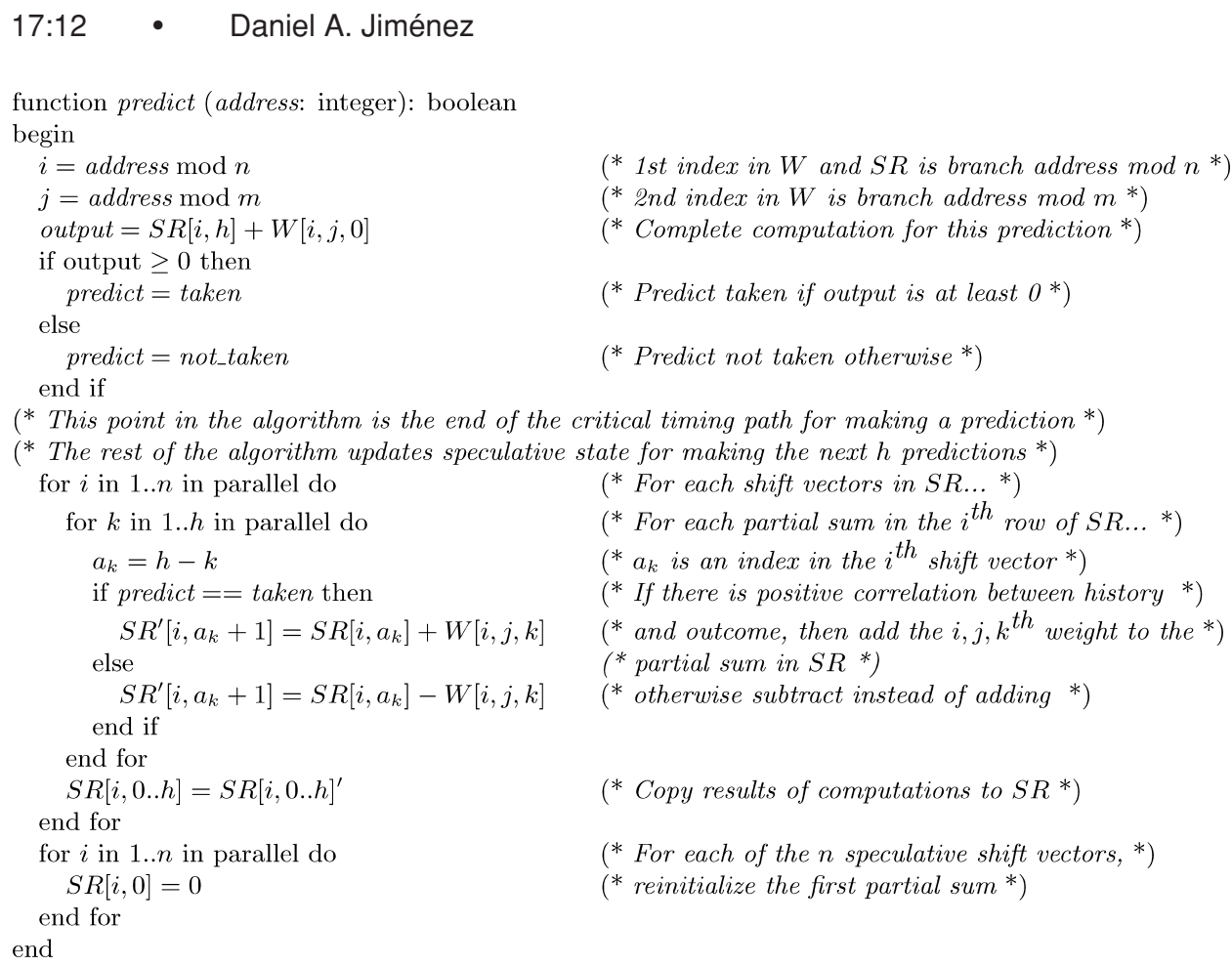

Fig. 6. Ahead-pipelined prediction algorithm.

enter the queue as 0 , are added to while in the queue, and on exiting are added to the bias weight and used to compute the prediction. $S R$ is speculative, since it assumes the correctness of predictions for unresolved branches. $R$ is a duplicate of $S R$ that is maintained nonspeculatively when a branch is resolved so that the predictor state can be restored on a misprediction. We have observed that 10 bits are sufficient for the elements of the $R$ and $S R$ arrays.

5.3.2 Making a Prediction. Figure 6 shows the new prediction algorithm for piecewise linear branch prediction with ahead-pipelining. To predict a branch that will occur $h$ branches in the future, the algorithm starts a partial sum for the output for that prediction with the value 0 . Each time a branch is predicted, its result is used to add another term to the partial sum to predict $h$ future branches. When a branch needs to be predicted, a partial sum is selected from one of $n$ candidate partial sums and added to the bias weight for that branch. Thus, the critical path for making a prediction is a table lookup, a multiplexer, and an addition. This incurs a delay comparable to other branch predictors, in particular the ahead-pipelined path-based neural branch predictor that is estimated to have a delay of from two to three cycles [Jiménez 2003].

5.3.3 Updating the Predictor. The algorithm for updating the predictor when a branch is executed is similar to the algorithm for the idealized predictor described in Section 3 with an extra step. The nonspeculative shift matrix $R$ 
is updated in the update phase using the outcome of the branch. Updating $R$ is similar to updating $S R$ except that the branch address and outcome are nonspeculative.

5.3.4 Misprediction Recovery. On a misprediction, the speculative contents of $S R$ must be overwritten with the nonspeculative version kept in $R$. This copying can be accomplished with low latency in parallel to other recovery activities taking place in the processor, such as restoring the register file from nonspeculative state. Indeed, we can think of the $n$ vectors in $S R$ as long registers in a special register file and apply known microarchitectural techniques to managing the speculative and nonspeculative versions of register files. In Section 8, we see that the size of $S R$ in terms of numbers of bits is no more than the size of a typical physical register file.

5.3.5 Implementation of Shift Matrix. It has been observed that partial sums for computing neural branch predictor outputs require no more than 11 bits to represent each partial sum [Jiménez 2003]. We find the 10 bits are sufficient for the piecewise linear branch predictor. Thus, an $n \times h$ shift matrix requires $n \times h$ 10-bit adders as well as $10 \times n \times h$ latches to store each partial sum. We propose an implementation that would use fast ripple-carry adders (RCA), which provide the best area and delay tradeoff for small bit widths [Eriksson et al. 2002].

We choose an RCA implementation that, scaling for $90 \mathrm{~nm}$ technology, consumes approximately the same area as 37 bytes in a single-ported $256-\mathrm{KB}$ tagless cache memory [Eriksson et al. 2002] when estimated using CACTI 3.0 [Shivakumar and Jouppi 2001]. This is a conservative estimate for converting adder area to memory area, since the memories we consider for storing branch predictor state have less favorable area characteristics than a $256-\mathrm{KB}$ memory. We take this extra area, as well as the latch area, into account when reporting results in Section 8 . That is, we allocate a certain number of bytes for each predictor, then take away 37 bytes from the piecewise linear branch predictor for each ripple-carry adder it consumes.

5.3.6 Implementation of $W$. Weights for neural branch prediction require no more than 8 bits to provide high accuracy [Jiménez and Lin 2002; Jiménez 2003]. The path-based neural predictor proposed using $h+1$ independently addressable tagless memories, each 8 bits wide, to implement the $W$ matrix. For the piecewise linear predictor, we propose using $h+1$ independently addressable tagless memories that are arranged as $n$ 8-bit words wide. That is, $W$ is organized as $h+1$ memories with $n$ blocks each with $m$ bytes. Thus, the training algorithm can update in parallel each of the $h+1$ weights responsible for predicting the branch.

5.3.7 Implementation of Parallel Algorithm. The prediction algorithm requires a large number of operations to occur in parallel. On each cycle, the speculative shift matrix receives $n \times h$ results in parallel, so $n \times h 10$-bit adders are required. For example, our most aggressive predictor design has $n=8$ and $h=51$, so it would require 40810 -bit adders. 
The nonspeculative version of the shift matrix, $R$, may be filled from the contents of the speculative version for right-path branches whose outcomes become known. This technique obviates the need for a second set of adders to maintain nonspeculative results.

Each byte of a block in each of the $h+1$ independently addressable memories corresponds to a different combination of lower address bits. Accessing the entire block once provides input to every iteration of the 1..n loop in the algorithm for making a prediction. Thus, each memory is accessed only once for each prediction, and once again for each update.

In the prediction phase of the algorithm, the $n$ shift vectors (i.e., the shift matrix) involved in computing the partial sums are formed by indexing each of the memories with the lower bits of the branch address and using the $n$ resulting bytes to form the corresponding $n$ elements of the shift matrix. In the updating phase of the algorithm, when $h+1$ elements from distinct weights vectors of one of the $n$ sets are updated, the implementation would write back entire blocks rather than single bytes, keeping the weights not modified the same.

\section{PIECEWISE LINEAR BRANCH PREDICTION IS A GENERALIZED NEURAL PREDICTOR}

A happy consequence of parameterizing piecewise linear branch prediction with $n$ and $m$ is that the new predictor becomes a generalization of concepts found in previous neural predictors. The perceptron predictor [Jiménez and Lin 2001] and path-based neural prediction [Jiménez 2003] turn out to be extreme ends of a family of parameterized piecewise linear branch predictors. Thus, piecewise linear branch prediction unifies previously distinct predictors and allows them to be studied in the same conceptual framework.

\subsection{With $m=1$, Piecewise Linear Prediction is the Perceptron Predictor}

The perceptron predictor keeps an array of $n$ weights vectors, each with $h+1$ integer weights. One weight in each vector is a bias weight and the other $h$ track correlation with branch outcome pattern history [Jiménez and Lin 2001]. When a branch is predicted, the branch address modulo $n$ is used to select a weights vector that is then used to compute the output of the predictor as the dot product of the weight vector and the branch history register.

In the piecewise linear branch predictor, if we let $m=1$, then every bit in the $G A$ array is 0 . The resulting piecewise linear branch predictor is equivalent to a perceptron predictor with $n$ perceptrons and a global history length of $h$. This is because letting $m=1$ effectively removes the second index of $W$, so $W$ collapses into a matrix whose $n$ rows represent perceptron weights vectors and whose $h+1$ columns correspond to the bias weights and weights correlating with branch outcome pattern history.

\subsection{With $n=1$, Piecewise Linear Branch Prediction is Path-Based Neural Branch Prediction}

The path-based neural predictor keeps an array of $m$ weights vectors, each with $h+1$ integer weights, again a bias and $h$ weights to correlate with history. 


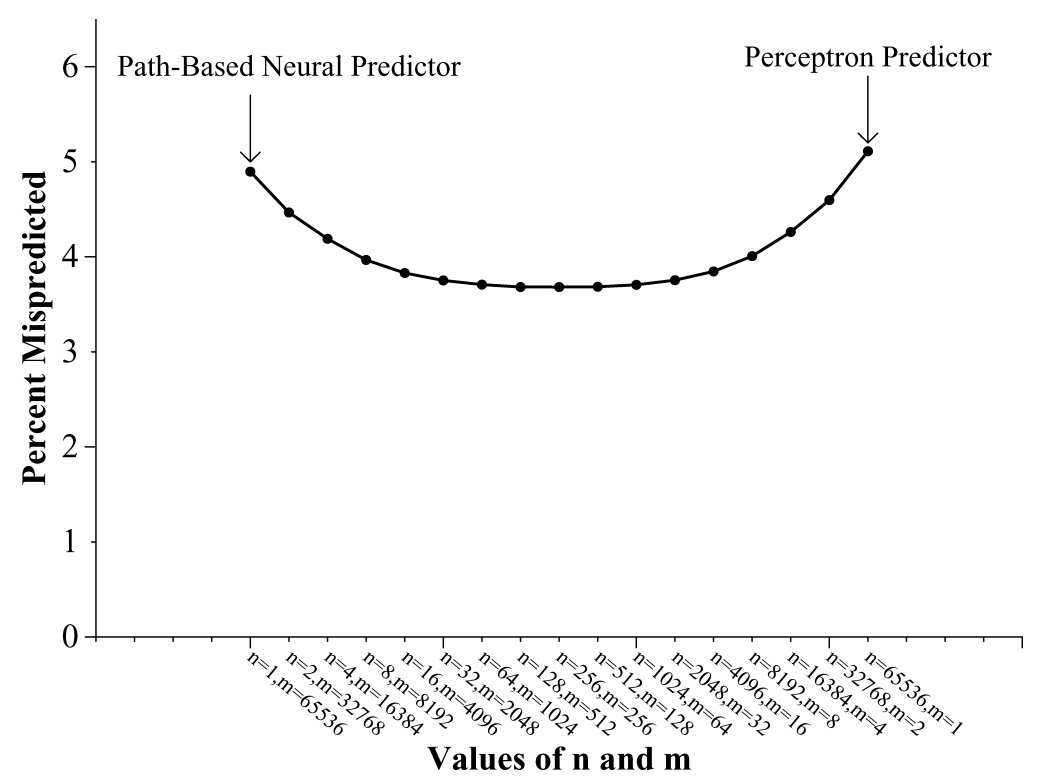

Fig. 7. Misprediction rates with values of $n$ and $m$ whose product is a constant $64 \mathrm{~K}$.

However, unlike the perceptron predictor, the path-based neural predictor uses the path history to select weights from up to $h+1$ distinct weights vectors for each prediction [Jiménez 2003].

In the piecewise linear branch predictor, letting $n=1$ effectively removes the first index of the $W$ and collapse $W$ into an $m \times(h+1)$ matrix of weights. The $m$ rows correspond to the $m$ weights vectors of the path-based neural predictor. The first column again corresponds to the bias weights for each branch address modulo $\mathrm{m}$. The rest of the $h$ columns correspond to the correlating weights chosen by path history. Since $n=1$, only the global path history is used to choose weights to make a prediction, which is just what path-based neural prediction does.

\subsection{Illustration of Generalized Predictor}

Figure 7 shows the results of experiments performed using our limit study infrastructure for piecewise linear branch predictors with a constant history length of $h=63$ and varying $n$ and $m$ such that their product is always 65,536. Thus, the number of elements in the $W$ array is kept at a constant $64 \times 65,536=$ $4 \mathrm{MB}$, but the first and second dimensions of the $W$ array are varied to represent different points in the space of neural predictors. The misprediction rates are averaged across all 15 benchmarks.

At the left end of the graph, we have $n=1$ and $m=65,536$. This predictor is equivalent to path-based neural prediction with $64-\mathrm{K}$ weights vectors. It achieves a misprediction rate of $4.9 \%$. At the right end of the graph, we have $n=65,536$ and $m=1$, equivalent to a perceptron predictor with 64 -K weights vectors. It achieves a misprediction rate of $5.1 \%$. In between these extrema are different configurations of piecewise linear branch predictors. Each of them 
outperforms both the perceptron predictor and path-based linear predictor. Thus, the neural predictors so far presented in the literature are the two worstcase examples of a better predictor! The minimum misprediction rates are slightly below 3.7\%, achieved when $n$ and $m$ are both between 128 and 512 . With a value of $n=8$ suitable for ahead-pipelined implementation, the misprediction rate is $3.9 \%$.

\section{METHODOLOGY FOR PERFORMANCE RESULTS}

This section describes the experimental methodology for obtaining simulated results for the realistic, ahead-pipelined version of piecewise linear branch prediction using a cycle-accurate simulator.

\subsection{Microarchitectural Framework}

We use 15 SPEC CPU integer benchmarks running under MASE/Alpha [Larson et al. 2001], a significantly modified version of SimpleScalar/Alpha [Burger and Austin 1997], a cycle-accurate out-of-order execution simulator that has been enhanced to include our branch predictors and to simulate overriding predictors at various latencies. We simulate all of the SPEC CPU 2000 integer benchmarks, and all of the SPEC CPU 95 integer benchmarks that are not duplicated in SPEC CPU 2000, except for 130.1i and 124.m88ks im because they would not work under our checkpoint-based simulation framework. The benchmarks are compiled with the CompaQ GEM compiler with the optimization flags -fast -04 -arch ev6.

We use SimPoint 1.1 to identify regions of the execution of each benchmark that characterize the entire run of the program on a given input. By analyzing statistics gathered from a functional simulation of the entire run of a benchmark on a given input, SimPoint finds simulation points, that is, regions of 100 million instruction executions [Sherwood et al. 2002]. We simulate these regions with MASE, recording statistics such as instructions-per-cycle rates and branch misprediction rates. We then aggregate these numbers in a weighted average to give a precise estimate of what the statistics would have been if the benchmarks had been run to completion.

We use the checkpointing facility of MASE to record the state of the simulated machine for each simulation point, thus avoiding the need to fast-forward the machine to the specified instruction for each simulation point. This methodology replaces earlier techniques of simulating only the first few hundred million instructions or using reduced inputs sets, both of which are unsatisfactory for estimating the performance of microarchitectures on long-running benchmarks, such as SPEC CPU.

Table I shows the reference input used for each benchmark.

Table II shows the base microarchitectural parameters used for the simulations. We started with a configuration loosely based on the Intel Pentium 4, but with an issue width of 16 and a deeper pipeline of 40 stages to provide a reasonable model of a future aggressively clocked microarchitecture. A recent study from Intel's Pentium Processor architecture group concludes that performance of aggressively clocked microarchitectures continues to improve until pipelines 
Table I. Inputs and Simulation Points for the SPEC CPU Integer Benchmarks

\begin{tabular}{|l|l|c|}
\hline Benchmark & \multicolumn{2}{|c|}{ Input } \\
\hline 099.go & $50 \times 21$ board & 1 \\
\hline 129.compress & bigtest.in & 4 \\
\hline 132.ijpeg & penguin.ppm & 3 \\
\hline 164.gzip & ref.graphic 60 & 4 \\
\hline 175.vpr & ref.net ref.arch.in & 4 \\
\hline 176.gcc & 166.i & 7 \\
\hline 181.mcf & ref.in & 5 \\
\hline 186.crafty & ref.in & 2 \\
\hline 197.parser & ref.in & 10 \\
\hline 252.eon & ref (cook) & 3 \\
\hline 253.perlbmk & makerand.pl & 4 \\
\hline 254.gap & ref.in & 4 \\
\hline 255.vortex & ref1.raw & 4 \\
\hline 256.bzip2 & ref.graphic 58 & 7 \\
\hline 300.twolf & ref & 4 \\
\hline
\end{tabular}

Table II. Microarchitectural Parameters

\begin{tabular}{|l|c|}
\hline Parameter & Configuration \\
\hline L1 I-cache & $16 \mathrm{~KB}, 64 \mathrm{~B}$ blocks, 2-way \\
\hline L1 D-cache & $16 \mathrm{~KB}, 64 \mathrm{~B}$ blocks, 4-way \\
\hline L2 unified cache & 1MB, 128B blocks, 4-way \\
\hline BTB & 4096 entry, 2-way \\
\hline Fetch/Decode/Issue/Commit & 16 wide \\
\hline Pipeline depth & 40 \\
\hline Reorder buffer size & 512 \\
\hline LSQ entries & 128 \\
\hline L2 hit latency & 7 cycles \\
\hline L2 miss latency & 500 cycles \\
\hline
\end{tabular}

reach a depth of 52 [Sprangle and Carmean 2002]. Since that study was presented, Intel has increased the depth of its Pentium 4 pipeline from 20 to 31 stages in a microprocessor named Prescott, available for purchase as of this writing. Thus, while our 40-stage pipeline is aggressive for current technology, it is conservative with respect to what is possible in future technologies.

We simulate extra pipeline stages beyond the five provided by sim-outorder by adding extra stages that simply buffer instructions from the fetch stage to the decode stage. Thus, our modeling of wrong-path effects is conservative with respect to branch prediction studies, since misspeculated loads that miss in the data cache can only be issued near the final stage.

\subsection{Branch Predictors Simulated}

We simulate the same predictors used in the limit study that appears earlier in this article. We simulate realistic, resource-bounded versions of $2 \mathrm{Bc}$-gskew, a global/local perceptron predictor, the path-based neural predictor, and the piecewise linear predictor. Since each predictor has a certain delay associated with it, even with ahead-pipelining, we use a two-level overriding organization [Jiménez et al. 2000] to mitigate predictor latency: A first-level 2-K-entry bimodal predictor gives a prediction in a single cycle and instructions are 
Table III. Tuned History Lengths for the Predictors and Values of $n$ and $m$ for Piecewise Linear Prediction

\begin{tabular}{|c|c|c|c|c|c|c|}
\hline \multirow{2}{*}{$\begin{array}{c}\text { Hardware } \\
\text { Budget }\end{array}$} & & Global/Local & Path-based & \multicolumn{3}{|c|}{ Piecewise Linear } \\
\cline { 5 - 7 } & 2Bc-gskew & perceptron & Neural & $h$ & $n$ & $m$ \\
\hline \hline $4 \mathrm{~KB}$ & 10 & $34 / 12$ & 19 & 19 & 1 & 215 \\
\hline $8 \mathrm{~KB}$ & 11 & $34 / 12$ & 19 & 19 & 2 & 176 \\
\hline $16 \mathrm{~KB}$ & 14 & $38 / 14$ & 24 & 23 & 4 & 138 \\
\hline $32 \mathrm{~KB}$ & 15 & $40 / 14$ & 31 & 26 & 8 & 118 \\
\hline $64 \mathrm{~KB}$ & 16 & $50 / 18$ & 34 & 43 & 8 & 151 \\
\hline $128 \mathrm{~KB}$ & 17 & $54 / 19$ & 38 & 50 & 8 & 288 \\
\hline $256 \mathrm{~KB}$ & 18 & $64 / 23$ & 40 & 51 & 8 & 603 \\
\hline
\end{tabular}

fetched down the predicted path. If the second-level predictor disagrees with the initial prediction, the instructions fetched so far are dropped and fetching continues from the other path. We also simulate an oracle branch predictor that always predicts correctly as well as the idealized version of piecewise linear branch prediction.

\subsection{Tuning The Predictors}

Using the train inputs of the benchmarks along with SimPoint and trace-driven simulation, we find the history lengths that minimize the average misprediction rate for each hardware budget and branch predictor. We use these history lengths in the execution-driven simulations on the ref inputs. For 2Bc-gskew, we test history lengths exhaustively, keeping the lengths that results in the lowest aggregate misprediction rate. For the global/local perceptron predictor, we exhaustively tune the global history, keeping the local history at a constant 10 and the percentage of the hardware budget allocated to the local history tables at approximately 35\%. Keeping the best global histories, we then tune the local histories exhaustively. For the path-based neural predictor, we tune the history length exhaustively. We use the formula $\theta=2.14(h+1)+20.58$ to set the threshold parameter $\theta$ in the path-based neural algorithm. We found this formula to give optimal accuracy at all history lengths in previous research.

For piecewise linear branch prediction, we tune history length as well as $n$ and $m$, the moduli for the first and second indices of the $W$ array, exhaustively. However, we constrain $n$ to be a power of two such that the number of bits required to store each of the $S R$ and $R$, matrices, that is, $10 \times n \times h$, never exceeds 4,096. Thus, restoring the $S R$ matrix from the $R$ matrix is comparable to restoring a register file of 32 64-bit registers after a mis-speculation. We use the same formula for $\theta$ as for the path-based neural predictor. Table III shows the tuned history lengths for each hardware budget for each predictor as well as values for $n$ and $m$ for the piecewise linear predictor. For many of the neural predictors, the chosen history lengths and hardware budgets would lead to a number of weights vectors that is not a power of 2 . We assume that a real design would use a power of 2 number of weights vectors for easy implementation. We present results with these configurations so that they may be compared against one another and against traditional table-based branch predictors using the same amount of state.

ACM Transactions on Architecture and Code Optimization, Vol. 5, No. 4, Article 17, Pub. date: March 2009. 
Table IV. Estimated Access Latencies

\begin{tabular}{|c|c|c|c|c|}
\hline $\begin{array}{c}\text { Hardware } \\
\text { Budget }\end{array}$ & $\begin{array}{c}\text { 2Bc-gskew } \\
\text { (cycles) }\end{array}$ & $\begin{array}{c}\text { Global/Local } \\
\text { Perceptron }\end{array}$ & $\begin{array}{c}\text { Path-based } \\
\text { Neural }\end{array}$ & $\begin{array}{c}\text { Piecewise } \\
\text { Linear }\end{array}$ \\
\hline \hline $4 \mathrm{~KB}$ & 2 & 5 & 2 & 2 \\
\hline $8 \mathrm{~KB}$ & 2 & 6 & 2 & 2 \\
\hline $16 \mathrm{~KB}$ & 2 & 6 & 2 & 2 \\
\hline $32 \mathrm{~KB}$ & 2 & 6 & 2 & 3 \\
\hline $64 \mathrm{~KB}$ & 3 & 7 & 3 & 3 \\
\hline $128 \mathrm{~KB}$ & 3 & 7 & 3 & 3 \\
\hline $256 \mathrm{~KB}$ & 3 & 7 & 3 & 4 \\
\hline
\end{tabular}

\subsection{Estimating Branch Predictor Latency}

We use CACTI 3.0 [Shivakumar and Jouppi 2001] to estimate the latencies of the various memories accessed by the predictors. We use HSPICE along with a custom logic design program to estimate the latency of the circuits used to compute the perceptron output for the perceptron predictor, the path-based neural predictor, and piecewise linear prediction.

Table IV shows the latencies we derived for each branch predictor and hardware budget giving the number of cycles it takes from the time a branch address is known to the time a prediction becomes available. We do not include the time it takes to compute an array index modulo $n$ or $m$; again, in an actual implementation, we would expect that these figures would be powers of 2 making the modulus operation a simple mask, but in this study, we allow $m$ to not be a power of 2 to facilitate comparison of predictors with the same amounts of state.

For 2Bc-gskew, we estimate the latency of the predictor as the delay in accessing the slowest table plus one fan-out-of-four (FO4) delay for taking the majority and choosing the hybrid prediction from the two component predictions.

For the global/local perceptron predictor, the latency is the sum of the access delay to the table of weights vectors measured by CACTI and the worst-case delay of the perceptron output circuit as measured by HSPICE. We optimistically ignore the access time to the first-level table of per-branch histories.

For the path-based neural predictor, the latency is the sum of the access delay to the table of bias weights and the worst-case delay of the adder that adds a bias weight to the next partial sum.

For the piecewise neural predictor, we compute the latency as the maximum of the access delay to the memory holding the bias weights for the current branch and the latency from reading $n$ partial sums from the $S R$ register. Then, we add a multiplexer delay for choosing a prediction from one of the $n$ partial sums and an adder delay for adding the selected partial sum to the bias weight. All of the estimates assume a $90 \mathrm{~nm}$ technology and an aggressive 8 FO4 delays, that is, $3.86 \mathrm{GHz}$.

\section{RESULTS FROM MICROARCHITECTURAL SIMULATION}

This section presents results of detailed microarchitectural simulation. Our main results compare the practical versions of piecewise linear branch 


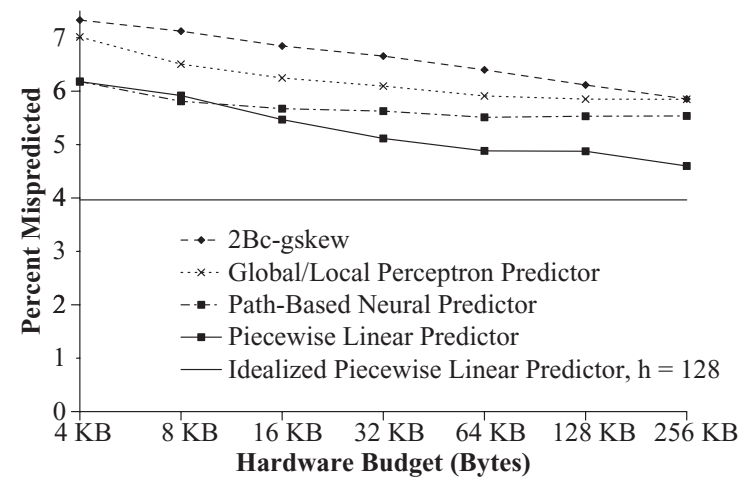

Fig. 8. Average misprediction rates per hardware budget.

prediction against practical versions of other branch predictors. We characterize the performance of these predictors using misprediction rates as well as instructions-per-cycle (IPC).

\subsection{Misprediction Rates}

Figure 8 shows the arithmetic mean misprediction rate of each predictor over all 15 benchmarks at hardware budgets from $4 \mathrm{~KB}$ to $256 \mathrm{~KB}$. It also shows the misprediction rate using the same cycle-accurate simulation methodology for the idealized piecewise linear predictor with a history length of 128 . As the hardware budget is increased, the advantage of the piecewise linear predictor over the other predictors increases. On average, at a 32-KB hardware budget, piecewise linear prediction mispredicts $5.1 \%$ of the time. That is $9 \%$ more accurate than path-based neural prediction which mispredicts $5.6 \%$ of the time and is the most accurate of the other predictors. At an aggressive 256-KB hardware budget, piecewise linear prediction with a $4.6 \%$ misprediction rate is $16 \%$ more accurate than path-based neural prediction with a $5.5 \%$ misprediction rate, and $22 \%$ more accurate than $2 \mathrm{Bc}$-gskew with a $5.9 \%$ misprediction rate. It is important to note that this improvement represents a departure from previous improvements in branch prediction accuracy-while all the other predictors in Figure 8 seem to approach an asymptotic misprediction rate of about $5.5 \%$, piecewise linear prediction continues getting better. The idealized piecewise linear predictor with a history length of 128 achieves a misprediction rate of just below $4.0 \%$. As we can see from the graph, the practical versions of this predictor reach within $15 \%$ of this limit. Indeed, the misprediction rate of piecewise linear branch prediction with a $256-\mathrm{KB}$ hardware budget is closer to the idealized predictor's misprediction rate than it is to the other predictors' rates.

Figure 9 shows the misprediction rates for $256-\mathrm{KB}$ branch predictors broken down by benchmark. Piecewise linear branch prediction yields the best accuracy for every benchmark except for 176 .gcc, on which it achieves a $2.1 \%$ misprediction rate compared with a $2.0 \%$ misprediction rate for the global/local perceptron predictor. On 252. eon the improvement is particularly good; piecewise 


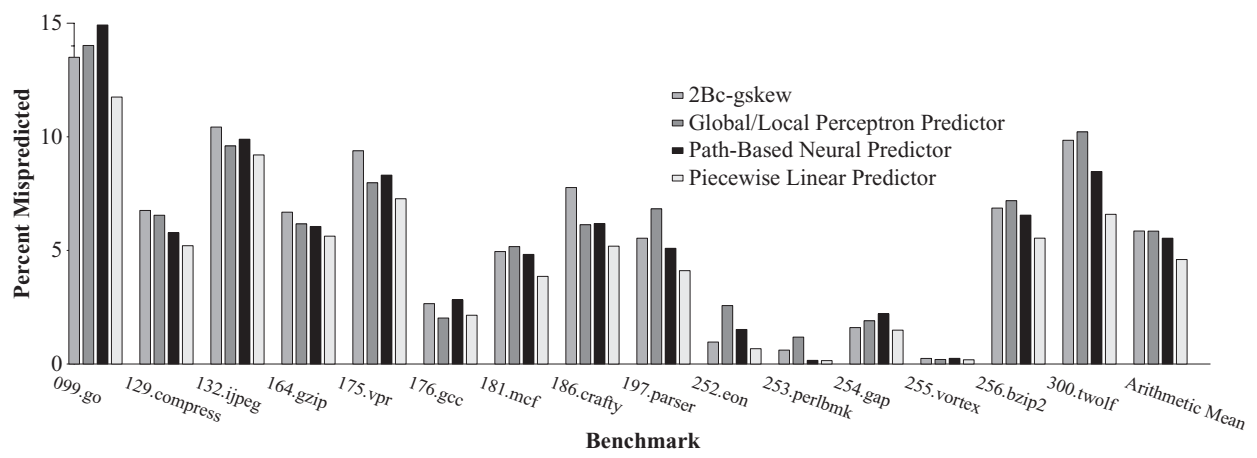

Fig. 9. Average misprediction rates per benchmark with a 256-KB hardware budget.

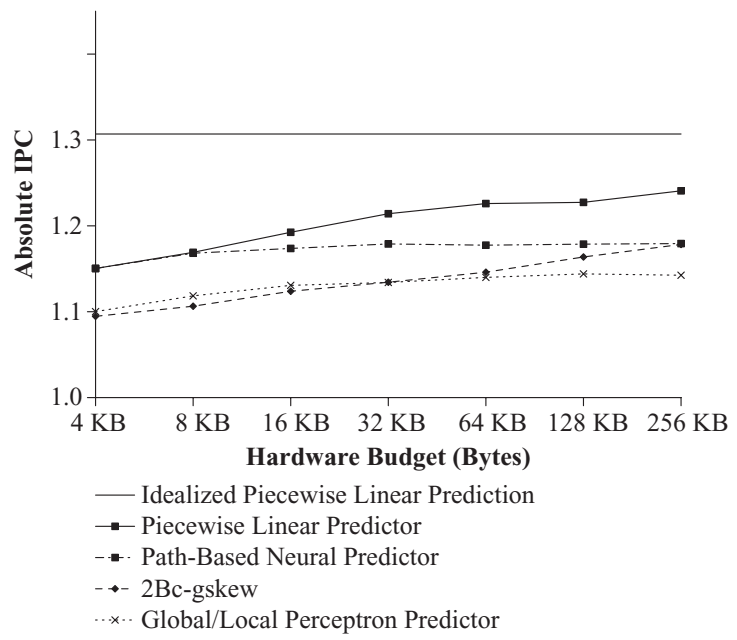

Fig. 10. Instructions per cycle.

linear prediction has a misprediction rate of $0.67 \%$, an improvement of $31 \%$ over $2 \mathrm{Bc}$-gskew at $0.97 \%, 56 \%$ over path-based neural prediction at $1.52 \%$, and $74 \%$ over the perceptron predictor at $2.57 \%$.

\subsection{IPC}

Figure 10 shows a graph giving the IPC for each predictor at hardware budgets ranging from $4 \mathrm{~KB}$ to $256 \mathrm{~KB}$. The graph shows the harmonic mean of the raw IPCs. Many factors independent of the branch predictor, such as locality, instruction mix, and the like, affect the IPCs of benchmarks with respect to one another. To isolate the effect of the branch predictor on performance, Figure 11 shows the harmonic mean of IPCs that have been normalized with respect to the IPC given by an oracle branch predictor that always predicts directions and targets correctly. Again, as with misprediction rates, the IPCs given by 


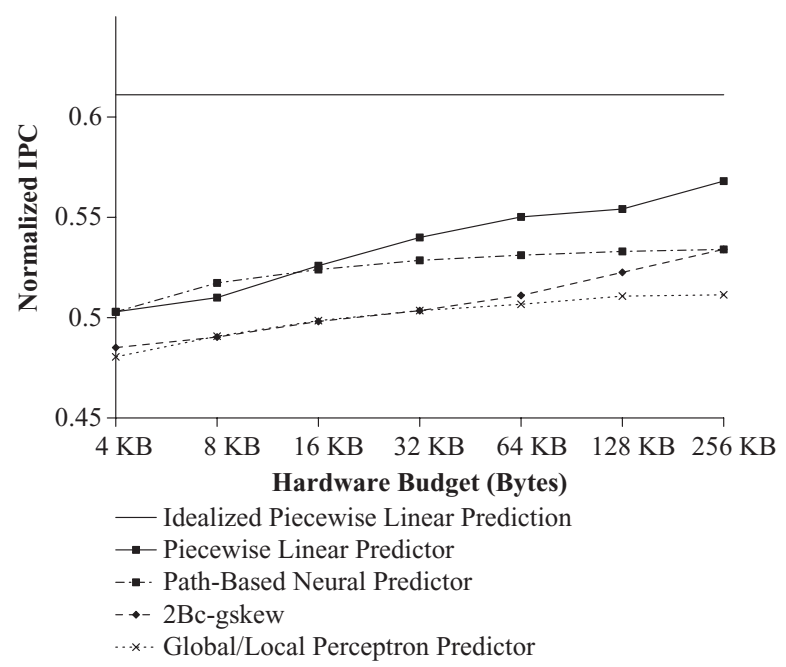

Fig. 11. Normalized IPC.

piecewise linear prediction improve with respect to the other predictors as the hardware budget increases. At a 16-KB hardware budget, piecewise linear prediction gives a speedup of $7 \%$ over $2 \mathrm{Bc}$-gskew.

Figure 13 shows the normalized IPCs for each benchmark using branch predictors with a hardware budget of $256 \mathrm{~KB}$. Piecewise linear branch prediction outperforms the other branch predictors on every benchmark except for 175 .vpr, for which $2 \mathrm{Bc}$-gskew yields a $2 \%$ speedup over piecewise linear prediction, most likely because of the higher latency of piecewise linear prediction compared with that of $2 \mathrm{Bc}$-gskew, and 176 .gcc. Although there is a significant variance among the IPCs given by the various branch predictors, all of them but piecewise linear branch prediction yield a harmonic mean normalized IPC of no more than 0.53 . Piecewise linear branch prediction gives a harmonic mean normalized IPC of 0.57 , a speedup of $8 \%$ over the other predictors. In the case of 186. crafty, piecewise linear branch prediction gives a speedup of $8 \%$ over path-based neural prediction, $20 \%$ over $2 \mathrm{Bc}$-gskew, and $15 \%$ over the perceptron predictor.

\subsection{Moderate Pipeline Depths}

Figure 12 shows the normalized IPCs achieved by simulating the various predictors for a machine with a more moderate pipeline depth of 20 stages. At a hardware budget of $64 \mathrm{~KB}$, piecewise linear branch prediction yields an improvement of $5 \%$ over $2 \mathrm{Bc}$-gskew and $4 \%$ over the path-based neural predictor. The gains are more modest because of reducing the pipeline depth reduces the penalty of mispredicted branches and also allow increases in the relative impact of branch predictor latency, which is highest for the piecewise linear branch predictor. 


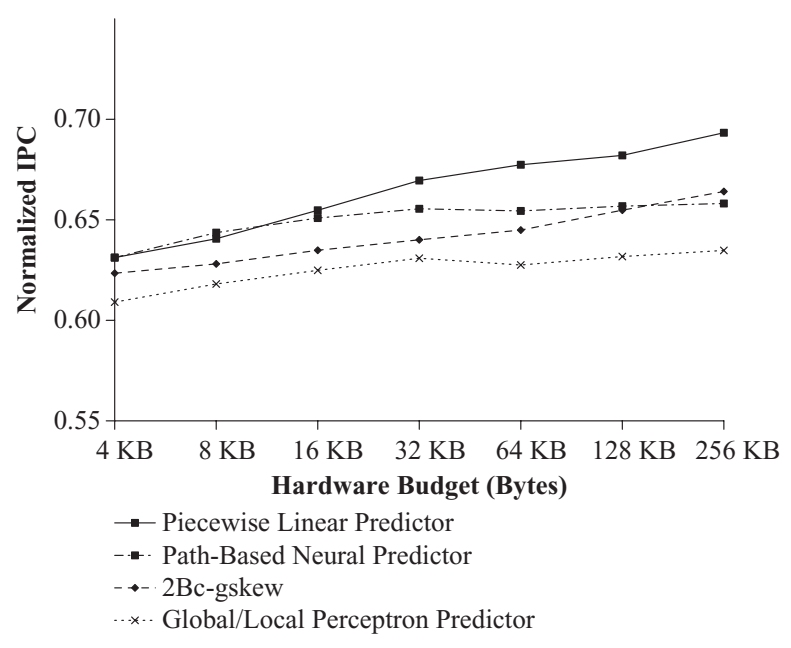

Fig. 12. Normalized IPC with moderate pipeline depth.

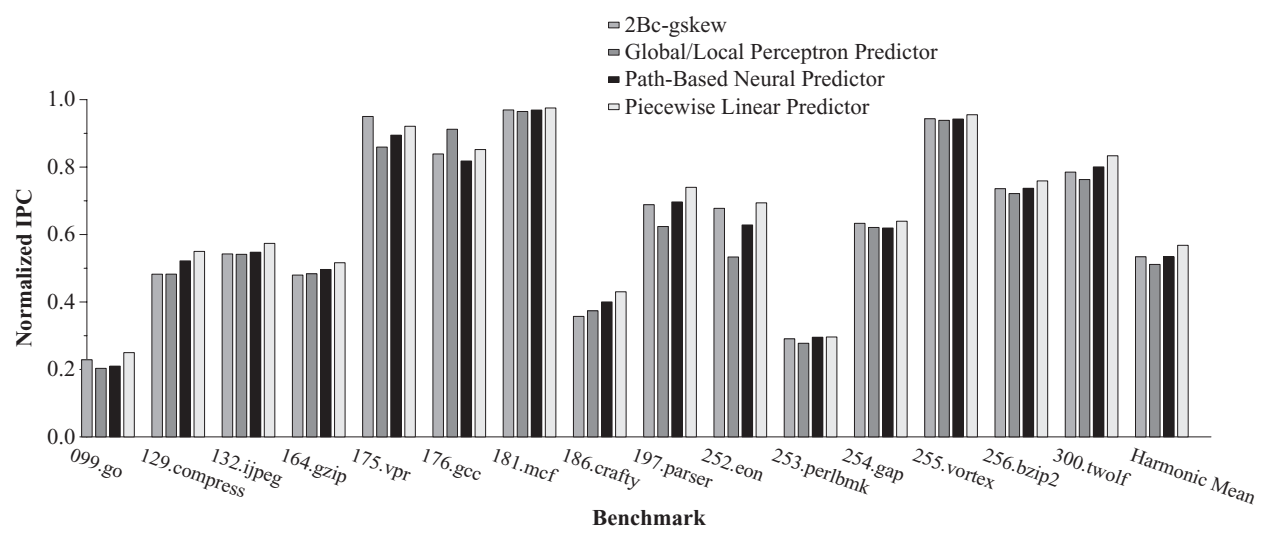

Fig. 13. IPC per benchmark with a hardware budget of 256-KB.

\section{ANALYSIS}

In this section, we explain why the piecewise organization is able to improve accuracy over the previous path-based and other global history branch predictors.

\subsection{Supporting Long Histories}

A central claim of the proponents of neural branch predictors has been that neural predictors allow longer global histories to be used. This is because traditional table-based predictors such as gshare, GAs, and 2Bc-gskew require resources exponential in history length, while the relationship between history length and resources is closer to linear for neural predictors [Jiménez and Lin 2002]. 
However, the history lengths that can be exploited by the original pathbased neural predictor are limited by destructive interference. Consider the first weight $w_{0}$ involved in a neural computation, coming $h$ branches before the branch to be predicted. As $h$ increases, the number of possible static branches that $w_{0}$ might help predict increases. More importantly, this number has a large variance, so some weights may help predict just a few branches and some will predict scores or hundreds, leading to a highly nonuniform distribution of interference between branches over the predictor's weights. The over-used weights either tend toward zero, contributing nothing to the prediction, or they accumulate noise, actively sabotaging the prediction.

Figure 4 from Section 4 illustrates this effect as well as the effect of longer histories on other branch predictors. As history length is increased from 1, all of the predictors yield significantly lower misprediction rates. However, all of the predictors except for piecewise linear yield a distinctive bowing curve that shows that they have an optimal history length that is shorter than the maximum history length tested. For 2Bc-gskew, the minimum misprediction rate is achieved at a history length of 34 . For the perceptron predictor, the best history length is 76 . At a length of 128 , the longest history tested, the perceptron predictor has a misprediction rate only $2 \%$ higher than its minimum.

For the path-based neural predictor, the best history length is only 56 . At the longest history of 128 , the misprediction rate is $14 \%$ higher than it is at 56 , due to the destructive interference inflicted on weights by multiple future static branches. Piecewise linear prediction with 32 sets eliminates this problem by incorporating address bits in the process of selecting the weights along the path to the branch to be predicted. The best history length for piecewise linear is the maximum of 128. Also, piecewise linear has a much lower misprediction rate in absolute terms than the other predictors. These results show that the original path-based neural predictor, while highly accurate when compared with other predictors, is still hobbled by the destructive interference that limits its ability to consider longer histories.

9.1.1 Analogy to GAg and GAs. The advantage of piecewise linear over path-based neural prediction is analogous to that of GAs over GAg. GAg, the simplest possible two-level branch predictor, only uses global pattern history to index the pattern history table (PHT) [Yeh and Patt 1993]. This results in a highly non-uniform access pattern to the PHT and thus massive destructive interference. GAs, which essentially uses address bits concatenated with history bits to index the PHT, has better accuracy than GAg because the distribution of accesses to the PHT is more uniform. (We discuss these predictors only because of their conceptual similarities to piecewise linear and path-based neural predictors. We do not compare experimentally against them because they are not as accurate as neural predictors or other predictors of the past several years.)

\subsection{Quantifying Aliasing}

Figure 14 shows graphs that characterize the destructive interference that plagues path-based neural prediction but not piecewise linear prediction. Each 

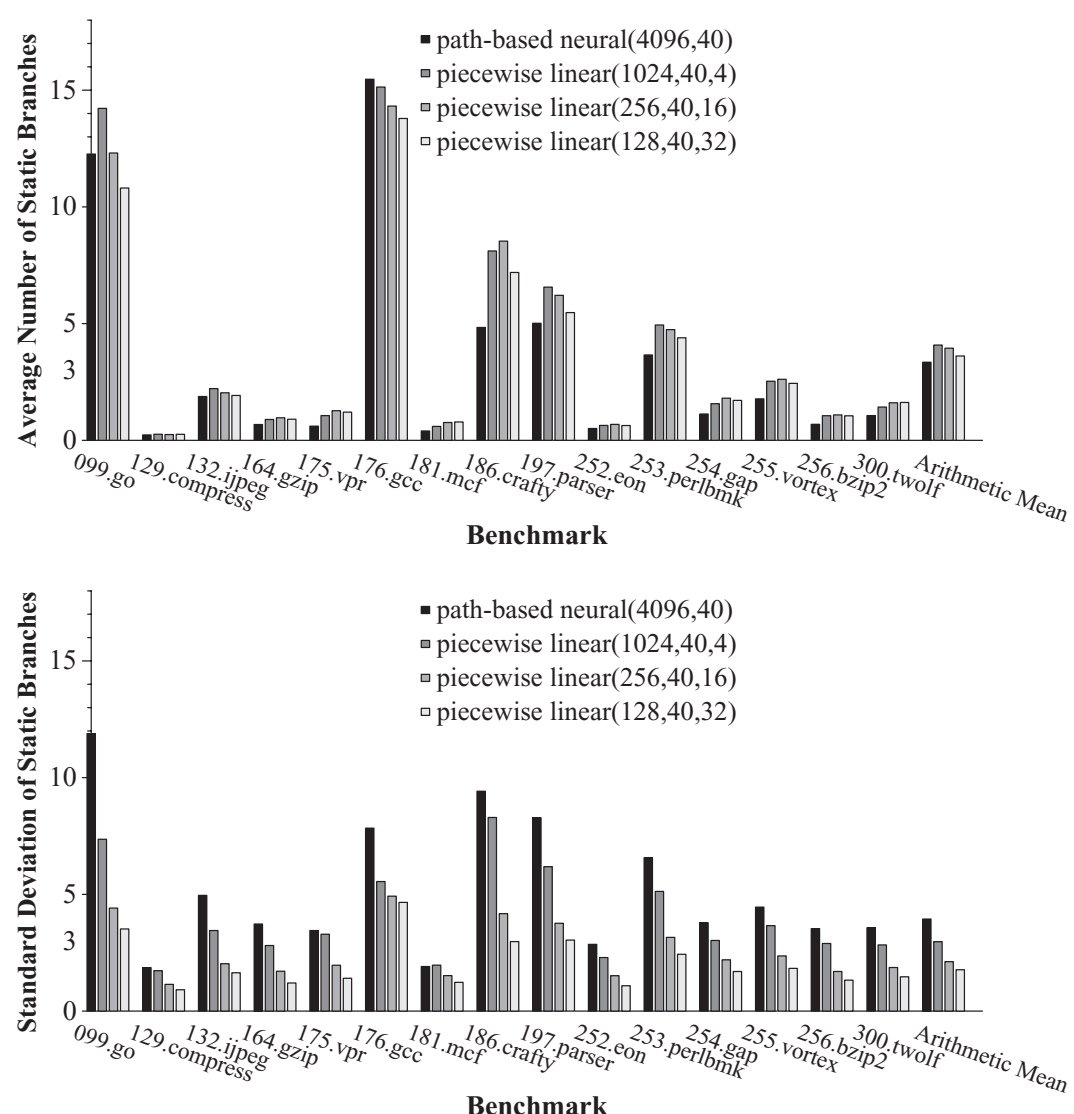

Fig. 14. Average and standard deviation of number of aliased static branches.

predictor measured uses a history length of 40 and the same hardware budget in terms of the number of bits of state used to represent the weights vectors. For the first graph, the $y$-axis shows, for the 40th weight in the average weights vector, the number of frequent static branches that weight will assist in predicting. (We consider a branch to be frequent with respect to a weight if it is responsible for at least $1 \%$ of the accesses to that weight.) This is a measure of the amount of interference to which the predictors are subjected. We can see that, on average, there is not a large difference in the average amount of interference between path-based neural and various versions of piecewise-linear.

However, the second graph shows the standard deviation of the number of static branches a weight will be used to help predict. Here, we can see that there is a high deviation of about four branches for path-based neural, while for piecewise-linear with 32 sets there is a low variance of about 1.8 branches. This means that some weights in path-based neural will be required to help predict many more static branches than others, while piecewise linear distributes the responsibility for prediction much more evenly over the weights, just as GAs provides a more even distribution of accesses to a PHT than GAg. 


\section{CONCLUSIONS AND FUTURE WORK}

Our practical piecewise linear branch prediction generalizes previous work on neural branch prediction. In doing so, it expands the design space of neural predictors giving rise to more accurate predictors that yield significantly better performance.

In future work, we plan to improve upon piecewise linear branch prediction by finding ways to reduce the extra hardware it requires and reduce the latency. We also plan to generalize this idea further by incorporating, for instance, perbranch history information into the piecewise linear predictor.

The normalized IPCs in Section 8 demonstrate that there is ample room for improvement in the performance delivered by branch predictors. We believe piecewise linear branch prediction will be a driving force for achieving this improvement.

\section{REFERENCES}

AkKary, H. AND SRINIVASAN, S. 2004. Using perceptron-based branch confidence estimation for speculation control. In Proceedings of the 10th International Symposium on High Performance Computer Architecture. IEEE, Los Alamitos, CA.

Block, H. D. 1962. The perceptron: A model for brain functioning. Rev. Mod. Phys. 34, 123-135.

Brekelbaum, E., Rupley, J., Wilkerson, C., And Black, B. 2002. Hierarchical scheduling windows. In Proceedings of the 35th International Symposium on Microarchitecture. IEEE, Los Alamitos, CA.

Burger, D. And Austin, T. M. 1997. The SimpleScalar tool set version 2.0. Tech. rep. 1342, Computer Sciences Department, University of Wisconsin.

Chen, I.-C. K., Coffey, J. T., AND Mudge, T. N. 1996. Analysis of branch prediction via data compression. In Proceedings of the 7th International Conference on Architectural Support for Programming Languages and Operating Systems. ACM, New York, 128-137.

Eriksson, H., Henriksson, T., and Larsson-Edefors, P. 2002. Full-custom vs. standardcell based design-an adder comparison. In Proceedings of the Swedish System-on-Chip Conference.

Evers, M., Patel, S. J., Chappell, R. S., and Patt, Y. N. 1998. An analysis of correlation and predictability: What makes two-level branch predictors work. In Proceedings of the 25th Annual International Symposium on Computer Architecture. IEEE, Los Alamitos, CA, 5261.

Falcón, A., Stark, J., Ramirez, A., Lai, K., and Valero, M. 2004. Prophet/critic hybrid branch prediction. In Proceedings of the 31st Annual International Symposium on Computer Architecture. IEEE, Los Alamitos, CA, 250.

JimÉNEZ, D. A. 2002. Reconsidering complex branch predictors. In Proceedings of the 9th International Symposium on High Performance Computer Architecture (HPCA-9). 43-52.

JIMÉNEZ, D. A. 2003. Fast path-based neural branch prediction. In Proceedings of the 36th Annual IEEE / ACM International Symposium on Microarchitecture (MICRO-36). IEEE, Los Alamitos, CA, 243-252.

JimÉNEZ, D. A. 2005. Idealized piecewise linear branch prediction. J. Instruct.-Level Parall. (JILP) 7 .

JimÉnez, D. A., Keckler, S. W., And Lin, C. 2000. The impact of delay on the design of branch predictors. In Proceedings of the 33rd Annual International Symposium on Microarchitecture (MICRO-33). IEEE, Los Alamitos, CA, 67-76.

JimÉnEZ, D. A. AND Lin, C. 2001. Dynamic branch prediction with perceptrons. In Proceedings of the 7th International Symposium on High Performance Computer Architecture (HPCA-7). IEEE, Los Alamitos, CA, 197-206.

JimÉnez, D. A. AND Lin, C. 2002. Neural methods for dynamic branch prediction. ACM Trans. Comput. Syst. 20, 4 , 369-397. 
Larson, E., Chatterjee, S., And Austin, T. 2001. MASE: A novel infrastructure for detailed microarchitectural modeling. In Proceedings of the International Symposium on Performance Analysis of Systems and Software. IEEE, Los Alamitos, CA.

LoH, G. H. AND Henry, D. S. 2002. Predicting conditional branches with fusion-based hybrid predictors. In Proceedings of the 11th Conference on Parallel Architectures and Compilation Techniques. ACM, New York, 165-176.

McFARLIng, S. 1993. Combining branch predictors. Tech. rep. TN-36m, Digital Western Research Laboratory.

Seznec, A. 2005. Genesis of the o-gehl branch predictor. J. Instruct.-Level Parall. 7.

Seznec, A., Felix, S., Krishnan, V., And Sazeides, Y. 2002. Design tradeoffs for the Alpha EV8 conditional branch predictor. In Proceedings of the 29th International Symposium on Computer Architecture. IEEE, Los Alamitos, CA.

Seznec, A. And Fraboulet, A. 2003. Effective ahead pipelining of instruction block address generation. In Proceedings of the 30th International Symposium on Computer Architecture. IEEE, Los Alamitos, CA.

Sherwood, T., Perelman, E., Hamerly, G., and Calder, B. 2002. Automatically characterizing large scale program behavior. In Proceedings of the 10th International Conference on Architectural Support for Programming Languages and Operating Systems. ACM, New York.

Shivakumar, P. AND Jouppi, N. P. 2001. Cacti 3.0: An integrated cache timing, power and area model. Tech. rep. 2001/2, Compaq Computer Corporation.

SpRANGle, E. AND CARMEAN, D. 2002. Increasing processor performance by implementing deeper pipelines. In Proceedings of the 29th International Symposium on Computer Architecture. IEEE, Los Alamitos, CA, 25-34.

Tarjan, D., Skadron, K., ANd Stan, M. 2004. An ahead pipelined alloyed perceptron with single cycle access time. In Proceedings of the Workshop on Complexity Effective Design (WCED).

YEH, T.-Y. AND PATT, Y. N. 1993. A comparison of dynamic branch predictors that use two levels of branch history. In Proceedings of the 20th Annual International Symposium on Computer Architecture. IEEE, Los Alamitos, CA.

Received August 2008; revised August 2008; accepted September 2008 